Methods The data on personnel, business activities, investments in work disability prevention and the costs of work disability were obtained from the companies and their occupational health care. The practices, structures, resources, and processes of work ability management, as well as internal and external co-operation were investigated through questionnaires and group interviews of the top management, HRM, supervisors, and employee representatives. We used the case descriptions, the complete mixed method data sets as a basis of the qualitative comparative analysis.

Results The companies increased investments into work disability prevention during follow-up by about $0.25 \%-1.5 \%$ of the payroll. Work disability costs per person-year decreased in about half of the participating companies and their units. In relation to payroll, the change in work disability costs in the studied organisations was $-2 \%$ to $1.5 \%$. From 2009 to 2013, and from 2010 to 2013, five and nine companies, respectively obtained a net benefit from their investments into work disability prevention. The reasons for the reduction in work disability costs were:

- the dismantling of barriers to co-operation,

- the visibility in practice of the strategic goals of work ability management,

- the way in which the work disability management measures focused on the main work disability risks, and

- the facilitation of multi-actor co-operation through coordination and the flow of information.

Discussion Companies that succeeded in managing changes and in maintaining the structures, activities and co-operation of these operations received more net benefits than those whose management of operations was disturbed by, for example, operational restructuring, or a significant person leaving HRM, the occupational safety organisation or occupational health services.

\section{EMPLOYER ACCOMMODATIONS AND PRODUCTIVITY LOSS FOR LONG-TERM SICK-LISTED WORKERS BEFORE, DURING AND AFTER SICK-LEAVE}

${ }^{1}$ Kerstin Ekberg* ${ }^{2}{ }^{2}$ Lars Bernfort, ${ }^{2}$ Jan Persson. ${ }^{1}$ Department of Medical and Health Sciences, Work and Rehabilitation, Linköping University, Sweden; ${ }^{2}$ Department of Medical and Health Sciences, Division of Health Care Analysis, Linköping University, Sweden

\subsection{6/oemed-2018-ICOHabstracts. 1552}

Introduction The study aimed to explore which interventions were performed by employers for sick-listed workers, employer costs for these measures, and if sickness presenteeism before and after the sick-leave period created costs for the employer.

Methods A web-questionnaire was sent to 393 supervisors of sick-listed workers (>30 days) due to common mental disorders or musculoskeletal disorders randomly drawn from the national AFA Insurance registers. The questionnaire comprised questions about work tasks, accommodations and interventions to facilitate RTW, and estimated costs for these measures. Presenteeism was measured by questions on duration and degree of reduced work performance before and after sick-leave. Lost productivity during sick-leave was based on a question regarding performance of replacements. Accommodations were grouped using factor analysis. Statistical analyses were performed with multiple logistic regression analysis.

Results Response rate was 50\% $(n=198)$. The most common interventions by supervisors were contacts with other stakeholders, most often HR, occupational health care, and/or Social Insurance Office. Changed work tasks were the most common interventions at the workplace and psychotherapy was the most common individual intervention. Supervisors had difficulties in estimating costs for these interventions. In multiple logistic regression analysis contacts with other stakeholders increased time to RTW $(\mathrm{OR}=0.68, \mathrm{p}<0.02)$, changed or increased staffing reduced time to RTW $(O R=1.43, p<0.05)$, as did ergonomic interventions $(\mathrm{OR}=1.74, \mathrm{p}<0.004)$, and work task adjustments $(\mathrm{OR}=1.41, \mathrm{p}<0.07)$. Demographic factors were not associated with time until RTW. Employer costs for productivity loss before, during and after sick-leave were on average 10000 EUR.

Discussion Workplace interventions reduce time to RTW, while supervisory contacts with other stakeholders are associated with prolonged sick-leave. Costs due to presenteeism before and after RTW highlights the need for increased attention to preventive measures at the workplace before sick-leave, as well as for support during re-integration after RTW, to reduce productivity loss.

\section{DEVELOPMENT AND PILOT TESTING OF A TRIAGE TOOL FOR SICKNESS ABSENCE COUNSELLING OF SICK-LISTED WORKERS WITHOUT AN EMPLOYMENT CONTRACT}

HJ van Rijssen*, JR Kim, M Roerdinkholder. Dutch Institute of Employee Benefit Schemes (UWV), Amsterdam, The Netherlands

\subsection{6/oemed-2018-ICOHabstracts. 1553}

Introduction Sick-listed workers without a (relative) permanent employment relationship, e.g. temporary agency workers and unemployed workers, are at higher risk for prolonged work disability compared to sick-listed employees. It is therefore important to start sickness absence counselling as early as possible. Triage - the process of prioritising patients on the base of the urgency of need for care - may facilitate this. The aim of this study was to develop and pilot test a triage tool to identify quickly the necessary intensity of counselling by het Social Security Agency (SSA) for sick-listed workers without an employment contract.

Methods Literature review, interviews and expert opinions were used to develop an initial triage tool. The developed tool consisted of a digital self-reported questionnaire for the worker and a (programmed) set of decision rules. Workers without an employment contract who were sick-listed between November 2016 and May 2017 at two locations of the Dutch SSA were requested to complete the questionnaire. Usability of the triage tool was evaluated by means of four group interviews with the occupational health care professionals of the SSA (e.g. sickness absence counsellors, insurance physicians).

Results The triage tool incorporated the workers perspective regarding the period of sickness absence, health status, their disability and several other risk factors. Approximately 5.300 sick-listed workers completed the questionnaire. The sickness 
absence counsellors were able to handle $27 \%$ of them without the direct assistance of an insurance physician. Results showed satisfaction with both the questionnaire and the decision rules among the professionals. Also, points of improvement were identified.

Conclusion This study indicates feasibility of the proposed triage tool, to effectively determine the necessary intensity of occupational health care interventions for sick-listed workers without an employment contract. To overcome important barriers and construct an optimal triage model, attention should be paid to further improve the questionnaire and decision rules.

\section{SUSTAINED RETURN TO WORK AFTER SICK LEAVE DUE TO MENTAL DISORDERS IN BRAZIL}

${ }^{1}$ JS Silva-Junior*, ${ }^{2} \mathrm{RH}$ Griep, ${ }^{3} \mathrm{MC}$ Martinez, ${ }^{4} \mathrm{FM}$ Fischer. ${ }^{1}$ National Social Security Institute, São Paulo, Brazil; ${ }^{2}$ Oswaldo Cruz Foundation, Rio de Janeiro, Brazil; ${ }^{3}$ WAF Informática, São Paulo, Brazil; ${ }^{4}$ School of Public Health, University of São Paulo, São Paulo, Brazil

\subsection{6/oemed-2018-ICOHabstracts.1554}

Introduction Several factors influence the effectiveness of labour reintegration after an episode of sick leave due to mental disorders (MD). When workers remain working more than 30 days after being back to work is known as sustained return-to-work (SRTW). This study aims to analyse factors associated to the effectiveness of S-RTW after sickness absence due to MD.

Methods A longitudinal study was conducted in the city of São Paulo, Brazil, from 2014-2016 and included 204 workers requiring sickness social security benefit due to MD. In baseline, participants fill questionnaires about sociodemographic info, health risk behaviours, work characteristics, health conditions and social security history. They were followed for 365 days after the first day of sickness absence. Those who have returned to work within this period were interviewed about employer's RTW process $(n=128)$. A multiple logistic regression was performed to analyse the factors that contributed to the S-RTW.

Result The average time for return to work was approximately six months among the $63.0 \%$ who tried to resume their work activities. The effectiveness rate among those who tried to return was 74\%. Factors associated to the S-RTW were: working for 5 or more years in the current job (HR 3.69; 95\% CI: 1.13 to 12.02 ), high return-to-work self-efficacy at baseline (HR 0.25; 95\% CI: 0.07 to 0.91 ) and was evaluated by a physician before RTW (HR 4.93; 95\% CI: 1.48 to 16.43) - when adjusted by sex, job title, depressive symptoms and social security benefit type.

Discussion Workers psychological conditions, occupational aspects and employer's RTW process are associated to the effectiveness of the workers reintegration to work. It is important to understand this process in order to encourage and to stimulate public and private intervention policies on tertiary prevention, focused in effective RTW after sick leave due to MD. Acknowledgment: CNPq for financial support (Grant $n^{\circ}$ 442051/2014-0).
550

WORKPLACE- AND SYSTEM-BASED INTERVENTIONS ON RETURN-TO-WORK AND RECOVERY FOR MUSCULOSKELETAL AND MENTAL HEALTH CONDITIONS: A SYSTEMATIC REVIEW

${ }^{1} \mathrm{KL}$ Cullen*, ${ }^{1} \mathrm{E}$ Irvin, ${ }^{2,3} \mathrm{~A}$ Collie, ${ }^{4} \mathrm{~F}$ Clay, ${ }^{5,6} \mathrm{U}$ Gensby, ${ }^{7} \mathrm{P}$ Jennings, ${ }^{1} \mathrm{~S}$ Hogg-Johnson, $1,8 \mathrm{~V}$ Kristman, ${ }^{9} \mathrm{M}$ Laberge, ${ }^{2} \mathrm{D}$ McKenzie, ${ }^{2} \mathrm{~S}$ Newnam, ${ }^{2} \mathrm{~A}$ Palagyi, ${ }^{2} \mathrm{R}$ Ruseckaite, ${ }^{10} \mathrm{D}$ Sheppard, ${ }^{4} \mathrm{~S}$ Shourie, ${ }^{11} \mathrm{I}$ Steenstra, ${ }^{1} \mathrm{D}$ Van Eerd, ${ }^{1,12} \mathrm{BC}$ Amick. ${ }^{1}$ Institute for Work and Health, Toronto, Canada; ${ }^{2}$ Institute for Safety Compensation and Recovery Research, Monash University, Melbourne, Australia; ${ }^{3}$ School of Public Health and Preventive Medicine, Monash University, Melbourne, Australia; ${ }^{4}$ mjury Research Institute, Monash University, Melbourne, Australia; ${ }^{5}$ National Centre for Occupational Rehabilitation, Rauland, Norway; ${ }^{6}$ Team WorkingLife ApS, Valby, Denmark; ${ }^{7}$ Dept. of Community Emergency Health and Paramedic Practice, Monash University, Melbourne, Australia; ${ }^{8}$ Lakehead University, Thunder Bay, Canada; ${ }^{9} \mathrm{CHU}$ Ste-Justine Research Centre, University of Montreal, Montreal, Canada; ${ }^{10}$ Accident Research Centre, Monash University, Melbourne, Australia; ${ }^{11} \mathrm{Ted}$ Rogers School of Management, Ryerson University, Toronto, Canada; ${ }^{12}$ Robert Stempel College of Public Health and Social Work, Florida International University, Miami, USA

\subsection{6/oemed-2018-ICOHabstracts. 1555}

Introduction The burden of managing musculoskeletal pain and injuries (MSDs) and mental health (MH) conditions in the workplace is substantial. While overall rates of work injury have declined in most high-income countries, there have not been equivalent improvements in RTW rates. The primary objective of this review was to synthesise evidence on the effectiveness of workplace- and system-based interventions for RTW and recovery after a period of work absence.

Methods We followed a systematic review process developed by the Institute for Work and Health and an adapted best evidence synthesis.

Result Seven electronic databases were searched from January 1990 until April 2015. This comprehensive search yielded 8898 non-duplicate references. Our synthesis identified 69 studies examining three types of RTW outcomes (lost time, work functioning and associated costs) and four recovery outcomes (pain, psychological functioning, physical functioning and quality-of-life). These studies examined interventions that were classified into three broad domains: healthcare provision, service delivery and workplace modifications. Our review identified that in most cases, interventions were multi-faceted and included multiple intervention components, sometimes operating across multiple domains. The most common RTW outcome reported was lost time. Among the other RTW and recovery outcomes, studies varied widely in their inclusion. There is strong evidence that interventions encompassing multiple domains are effective in improving RTW outcomes in workers with MSD or MH conditions. In contrast, there is moderate to strong evidence that most single-component interventions have no effect on improving recovery regardless of condition.

Discussion While there is substantial research literature focused on RTW, only a small percentage of these intervention studies also include measures of recovery. Identifying effective intervention programs that facilitate RTW and recovery allows workplaces to implement empirically supported programs that benefit workers through improved function and reduced pain while reducing the economic burden associated with lost time. 